\title{
Canine gastrointestinal tract tumours: a restrospective study of 74 cases
}

\author{
Lucia Frgelecová ${ }^{1}$, Miša Škorič ${ }^{1}$, Petr Fictum ${ }^{1}$, Roman Husník ${ }^{2}$ \\ ${ }^{1}$ University of Veterinary and Pharmaceutical Sciences Brno, Faculty of Veterinary Medicine, Department \\ of Pathological Morphology and Parasitology, ${ }^{2}$ Small Animal Clinic, Department of Internal Medicine, Brno, \\ Czech Republic
}

\begin{abstract}
The aim of our study was to investigate histopathologically the incidence of gastrointestinal tract tumours in dogs in the Czech Republic in relation to their age, sex and breed. In total, 29 gastric and 45 intestinal tumours from 3827 gastrointestinal tract biopsies were investigated. The average age of dogs with gastric lymphoma, adenocarcinoma, and other mesenchymal tumours was 7.3 years, 9.5 years, and $>10$ years, respectively. Breed predisposition to adenocarcinoma was evidenced in the Belgian Shepherd and Leonberger, to lymphoma in the Doberman $(P<0.01)$. The most frequent site of adenocarcinoma was antrum pyloricum (66.7\%); lymphomas involved gastric wall diffusely. The average age of dogs with intestinal adenoma, adenocarcinoma, lymphoma, leiomyoma or leiomyosarcoma and carcinoid was $7.5,8.5,8.5,12$, and 14 years, respectively. The age predisposition to intestinal adenoma and lymphoma was $7-8$ years $(P<0.01)$ and to adenocarcinoma $7-8$ years $(P<0.05)$; mesenchymal tumours occurred at the age of $11-12$ years $(P<0.01)$. Breed predisposition to intestinal adenoma was evidenced in the Pug, Leonberger and English Setter $(P<0.01)$, to adenocarcinoma in the English Setter $(P<0.01)$ and Hovawart $(P<0.05)$, to lymphoma in the Doberman and Hovawart $(P<0.05)$. The most frequent site of occurrence of intestinal tumours was the colorectal region. The study revealing breed, sex and age predisposition to gastrointestinal tumours in dogs has not been described yet in the literature.
\end{abstract}

Dog, neoplasia, histopathology, stomach, intestine, immunohistochemistry

Retrospective studies of gastrointestinal tract tumours in dogs are less common than case reports of these tumours. These tumours occur less commonly compared to tumours of other organ systems. The incidence of these tumours in dogs is rather higher than in other domestic animals. The occurrence of malignant tumours is, compared to benign variants, more frequent and, except for lymphomas, majority of them are classified as carcinomas.

In 2003, World Health Organization set out the Histological Classification of Tumors of the Alimentary System of Domestic Animals counting over 50 specific tumour types and over 15 additional pseudotumourous lesions of the gastrointestinal tract (Head et al. 2003). The age is considered as a risk factor, and majority of these tumours are evidenced in elderly animals. The highest incidence in dogs was recorded at the age of 8-9 years (Head et al. 2002). According to most of the studies, it seems that breed and sex do not play a significant role in the frequency of tumour incidence; however, some studies indicated a higher incidence of gastric carcinomas in male elderly dogs compared to females (Patnaik et al. 1977; Twedt 1992). Some authors also proved higher incidence of gastric carcinomas in some breeds of dogs such as Rough Collie, Staffordshire Bull Terrier and Belgian Shepherd (Sullivan et al. 1987; Fonda et al. 1989; Scanziani et al. 1991).

Intestinal adenocarcinomas in dogs represent approximately $0.5 \%$ of all malignant tumours and approximately $40 \%$ of them are located in the colon and rectum. The average age of dogs with intestinal adenocarcinoma is 9 years. According to majority of authors, sex does not represent a risk factor, though some sources indicate higher incidence in males with more frequent location of tumours in the duodenum, colon and rectum (Brown et al. 2007). The average age of dogs with primary intestinal lymphomas was recorded in most cases at 6.7 years, with the age interval from 19 months to 13 years, and with higher incidence in males (Brown et al. 2007). It seems that breed does not influence significantly

Address for correspondence:

Miša Škorič

Department of Pathological Morphology and Parasitology

Faculty of Veterinary Medicine

University of Veterinary and Pharmaceutical Sciences Brno

Palackého tř. 1/3, 61242 Brno, Czech Republic

Phone: $+420541562252,+420606812621$

E-mail: skoricm@vfu.cz

http://actavet.vfu.cz/ 
the frequency of intestinal carcinomas, although some authors found a higher incidence in the Boxer, Collie, Poodle and German Shepherd (Patnaik et al. 1977; Brown et al. 2007). Other types of gastrointestinal tract tumours are generally recorded less commonly.

The aim of this study was to investigate the incidence of gastrointestinal tract tumours in dogs in the Czech Republic in relation to their age, sex and breed.

\title{
Materials and Methods
}

\begin{abstract}
Materials for this study were collected from the biopsy files of the pathology department of the University of Veterinary and Pharmaceutical Sciences, Brno, Czech Republic for a 5-year period (2008-2012). A total number of 74 gastrointestinal tract tumours were diagnosed in 43 different breeds and 6 cross-breed dogs. Sex ratio in the diagnosed tumours was 43:31 in favour of males. Dogs were divided by age into 8 groups with the age span from 1 to 16 years. All available medical records were studied regarding age, sex and breed of affected dogs and sites of the tumours. Bioptical tissue samples were fixed in 10\% neutral buffered formalin, processed and stained with haematoxylin and eosin (HE) and immunohistochemistry was performed when needed. The following primary antibodies with their dilution were used: CD3 (1:50), CD79 (1:25), CD117 1:100), cytokeratin AE1/AE3 (1:50), vimentin (1:50), S-100 protein (1:200), glial fibrillary acidic protein (GFAP) (1:50), smooth muscle actin (SMA) (1:50) and F-VIII (1:100) (DAKO, Glostrup, Denmark); secondary antibody was the part of the detection system EnVision (DAKO).

The data were statistically analyzed with chi-square testing (program UNISTAT 5.6 for Excel); the results were significant when $P<0.01$ and $P<0.05$.
\end{abstract}

\section{Results}

In our study, we diagnosed 29 gastric $(0.8 \%)$ and 45 intestinal $(1.2 \%)$ tumours from the total number of 3,827 biopsies. The frequency of gastrointestinal tract tumours was $1.9 \%$ from all biopsies. Gastric adenocarcinoma was detected in 13 breeds (with a higher incidence in the Belgian Shepherd and Golden Retriever, lymphoma was detected in 6 breeds (with a higher incidence in the Doberman and Golden Retriever), adenoma was detected in 4 breeds, GIST in 2 and leiomyoma in one breed. Intestinal adenoma was detected in 15 breeds (with a higher incidence in the Pug and Maltese), adenocarcinoma in 7, lymphoma in 11 breeds (with a higher incidence in the English Cocker Spaniel), leiomyoma, leiomyosarcoma, GIST, haemangioma, ganglioneuroma, osteosarcoma and carcinoid singly.

Malignant tumours occurred more frequently in the stomach $(90 \%)$. The number of gastric tumours of epithelial origin $(60 \%)$ was higher than those of mesenchymal origin $(55 \%)$. In stomach, higher incidence of malignant epithelial tumours $(60 \%)$ was recorded compared to benign epithelial and mesenchymal tumours. Tables 1 and 2 show the incidence of gastric tumours in relation to age and sex. We evidenced breed predisposition to gastric lymphoma in the Doberman $(P<0.01)$ and breed predisposition to gastric adenocarcinoma in the Belgian Shepherd $(P<0.01)$ and Leonberger $(P<0.01)$. The most frequent site of gastric tumours was antrum pyloricum $(66.7 \%)$, followed by fundus ventriculi. Primary tumour location with the lowest frequency of incidence was angulus ventriculi and curvatura ventriculi major (6.7\%). Lymphomas involved the gastric wall diffusely.

In the intestines, higher incidence of benign epithelial tumours (40\%) was recorded; the frequency of malignant epithelial tumours was $20 \%$ and malignant tumours of mesenchymal origin represented $33.3 \%$. Tables 3 and 4 show the incidence of intestinal tumours related to age and sex. The most frequent site of intestinal tumours was the colorectal region $(66.7 \%)$, followed by the small intestine $(28.9 \%)$ and caecum $(4.4 \%)$.

A significant number of intestinal adenomas were detected in the colorectal region $(94.4 \%)$, and the most affected intestinal segment was the rectum. We recorded 12 adenomas in the rectum from the total number of $18(66.7 \%)$ colorectal tumours. Adenocarcinomas were recorded in the rectum $(55.6 \%)$ and colon $(33.3 \%)$. Intestinal lymphomas involved more segments concurrently. Rare intestinal tumours, such as ganglioneuroma and hemangioma, occurred in the rectum. In the caecum we detected leiomyosarcoma and gastrointestinal 
Table 1. Age distribution of dogs with a gastric tumour.

\begin{tabular}{|c|c|c|c|c|c|c|c|}
\hline \multirow{2}{*}{ Age (years) } & \multirow{2}{*}{ Biopsies } & \multirow{2}{*}{$\mathrm{n}$} & \multicolumn{5}{|c|}{ Type of gastric tumour (number of dogs with a tumour) } \\
\hline & & & A & $\mathrm{AC}$ & $\mathrm{L}$ & GIST & LS \\
\hline $1-2$ & 674 & 1 & & & & & 1 \\
\hline $3-4$ & 366 & & & & & & \\
\hline $5-6$ & 480 & 5 & & 2 & & & 3 \\
\hline $7-8$ & 573 & 7 & & 3 & & 1 & 3 \\
\hline $9-10$ & 529 & 5 & 1 & 4 & & & \\
\hline $11-12$ & 422 & 7 & & 4 & 1 & 1 & 1 \\
\hline $13-14$ & 350 & 4 & & 2 & 1 & & 1 \\
\hline $15-16$ & 148 & 0 & & & & & \\
\hline Unknown & 285 & 0 & & & & & \\
\hline Total & 3827 & 29 & 1 & 15 & 2 & 2 & 9 \\
\hline Mean age & & & 9 & 9.5 & 12.5 & 10 & 7.3 \\
\hline
\end{tabular}

A - adenoma, AC - adenocarcinoma, L - leiomyoma, GIST - gastrointestinal stromal tumour, LS - lymphosarcoma, $\mathrm{n}$ - number of dogs with gastric tumours

Table 2. Sex distribution of dogs with a gastric tumour.

\begin{tabular}{lccccccc}
\hline \multicolumn{7}{c}{ Type of gastric tumour (number of dogs with a tumour) } \\
\hline Sex & Biopsies & $\mathrm{n}$ & A & AC & L & GIST & LS \\
\hline Male & 1720 & 16 & & 8 & 2 & 1 & 5 \\
Female & 1994 & 13 & 1 & 7 & & 1 & 4 \\
Unknown & 113 & 0 & & & & & \\
\hline Total & 3827 & 29 & 1 & 15 & 2 & 2 & 9 \\
\hline
\end{tabular}

A - adenoma, AC - adenocarcinoma, L - leiomyoma, GIST - gastrointestinal stromal tumour, LS - lymphosarcoma, $\mathrm{n}$ - number of dogs with gastric tumours

stromal tumour, and the site of osteosarcoma was the jejunum. Intestinal epithelial tumours occurred with highest frequency in the colorectal region (92.6\%); lymphomas (11 cases) occurred diffusely in more segments of the small intestine.

Breed predisposition to intestinal adenoma was evidenced in the Pug $(P<0.01)$, Leonberger $(P<0.01)$ and English Setter $(P<0.01)$. Breed predisposition to intestinal adenocarcinoma was evidenced in the English Setter $(P<0.01)$ and Hovawart $(P<0.05)$. Breed predisposition to intestinal lymphoma was noted in the Doberman $(P<0.05)$ and Hovawart $(P<0.05)$. Carcinoid was recorded in the Dachshund, osteosarcoma in the Fila Brasileiro, ganglioneuroma in the Leonberger, gastrointestinal stromal tumour and leiomyosarcoma in the crossbreed and leiomyosarcoma in the Miniature Schnautzer.

\section{Discussion}

The frequency of gastrointestinal tract tumours counted $1.9 \%$ from all biopsies, of which $0.8 \%$ were gastric and $1.2 \%$ intestinal tumours. Patnaik (1977) recorded $1.1 \%$ of incidence of gastrointestinal tumours, of which $0.5 \%$ were gastric and $0.6 \%$ intestinal tumours. Other authors showed the incidence of these tumours up to $1 \%$ (Swan and Holt 2002) and up to 3\% (Russell et al. 2007). We found highly significant percentage of all gastric tumours represented by adenocarcinomas (93.8\%); higher incidence of malignant epithelial tumours was also confirmed by other authors (Sautter and 

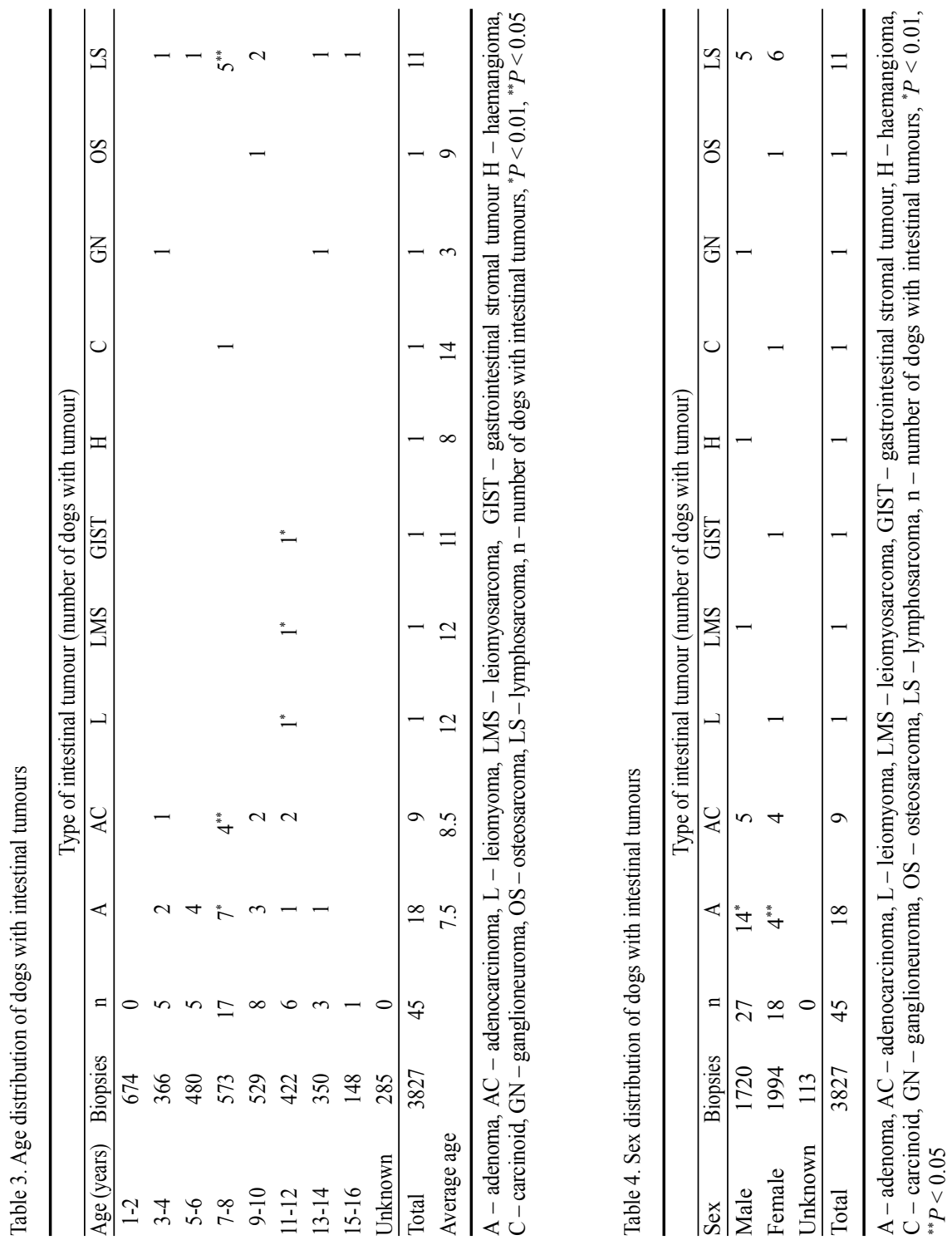

Hanlon 1975; Patnaik et al. 1977; Sullivan et al. 1987; Swan and Holt 2002). Some authors found higher incidence of gastric tumours in male dogs (Simpson 2010). In our study, the frequency of gastric tumours was 16:13 in favour of males and the average age of dogs with gastric adenocarcinoma was 9.5 years. Patnaik et al. (1977) and Simpson (2010) described the incidence of gastric carcinoma in dogs of similar ages of 9.5 and 10 years, respectively. In some studies, the frequency of gastric adenocarcinoma was recorded as significant in some breeds of dogs (Patnaik et al. 1977; Scanziani et al. 1991; Swann and Holt 2002; Simpson 2010). We followed 
also the concrete anatomical location of primary tumour occurrence in stomach and similar results were obtained by other authors (Patnaik et al. 1977; Swann and Holt 2002; Brown et al. 2007; Simpson 2010).

In our study, adenocarcinomas counted $1 / 3$ of intestinal tumours, located mainly in the colorectal region, and we evidenced age predisposition (7-8 years). Patnaik et al. (1977) and Hall and German (2010) recorded adenocarcinomas in dogs of the mean age of 9 years. In comparison with our results, some works described higher incidence of intestinal adenocarcinomas in males (Patnaik et al. 1977; Head et al. 2002; Brown et al. 2007). The most frequent mesenchymal tumour in our study was lymphoma, which corresponds to the results of Patnaik et al. (1977). They found lower frequencies $13.8 \%$ and $6.7 \%$ in other mesenchymal tumours in the stomach and intestine, respectively. The occurrence of these tumours in dogs is rather more frequent in the small intestine and colon than in the stomach (Brown et al. 2007; Gillespie et al. 2011). We detected 3 cases of gastrointestinal stromal tumours, with similar sex distribution as observed by other authors (Head et al. 2002; Brown et al. 2007). Some authors found higher incidence of leiomyosarcomas in females of the German Shepherd breed (Simpson 2010), with incidence at the mean age of $>10$ years (Patnaik et al. 1977; Cohen et al. 2003; Simpson 2010). These findings correspond with our results. In our study, the mean age was 12.5 years in dogs with gastric leiomyoma, 10 years in dogs with gastrointestinal stromal tumour, 12 years in dogs with intestinal leiomyoma and leiomyosarcoma, and gastrointestinal stromal tumour was recorded at the age of 11 years. Another study (Russell et al. 2007) showed the mean age of 10.4 years for gastrointestinal stromal tumours, with sex distribution 23:19 in favour of males. It seems that gastrointestinal stromal tumours in dogs showed different biological behavior in comparison to leiomyosarcomas. For the differentiation between gastrointestinal stromal tumours and leiomyosarcomas we used markers CD117, SMA, vimentin and S-100 protein, although some authors recommended using the other additional markers (Jelínek et al. 2009). The use of combination of these antibodies seems to be sufficient in diagnostics of tumours. In conclusion, this extensive retrospective study revealed some breed, sex and age predisposition to gastrointestinal tract tumours in dogs that has not been described in the literature yet.

\section{Acknowledgements}

We thank doc. RNDr. Iveta Bedáňová, Ph.D. for help with statistical analysis of our data.

\section{References}

Brown CC, Baker DC, Barker IK 2007: Neoplastic and proliferative lesions of the stomach and intestine. In: Grant Maxie M (ed.): Jubb, Kennedy \& Palmer's Pathology of Domestic Animals. Saunders Ltd., pp. 116-127 Cohen M, Post GS, Wright JC 2003: Gastrointestinal leiomyosarcoma in 14 dogs. J Vet Intern Med 17: 107-110

Fonda D, Gualtieri M, Scanziani E 1989: Gastric carcinoma in the dog: A clinicopathological study of 11 cases. J Small Anim Pract 30: 353-360

Gillespie V, Baer K, Farrelly J, Craft D, Luong R 2011: Canine gastrointestinal stromal tumors: Immunohistochemical expression of CD34 and examination of prognostic indicators including proliferation markers Ki67 and AgNOR. Vet Pathol 48: 283-291

Hall EJ, German AJ 2010: Diseases of the small intestine. In: Ettinger SJ, Feldman EC (eds.): Textbook of veterinary internal medicine, Saunders Elsevier, Canada, pp. 1526-1572

Head KW, Cullen JM, Dubielzig RR, Else RW, Misdorp W, Patnaik AK, Tateyama S, Van der Gaag I 2003: Histological classification of tumors of the alimentary system of domestic animals. Armed Forces Institute of Pathology, Washington, DC.

Head KW, Else RW, Dubielzig RR 2002: Tumors of the alimentary tract. In: Meuten DJ (ed.): Tumors in Domestic Animals. Iowa state Press, Iowa, pp. 451-477

Jelínek F, Hron P, Hozmanová F 2009: Gastrointestinal stromal tumour in a guinea pig: a case report. Acta Vet Brno 78: 287-291

Patnaik AK, Hurvitz AI, Johnson GF 1977: Canine gastrointestinal neoplasms. Vet Pathol 14: 547-555

Russell KN, Mehler SJ, Skorupski KA, Baez JL, Shofer FS, Goldschmidt MH 2007: Clinical and 
immunohistochemical differentiation of gastrointestinal stromal tumors from leiomyosarcomas in dogs: 42 cases (1990-2003). J Am Vet Med Assoc 230: 1329-1333

Sautter JH, Hanlon GF 1975: Gastric neoplasms in the dog: a report of 20 cases. J Am Vet Med Assoc 166: 691-696

Scanziani E, Giusti AM, Gualtieri M, Fonda D 1991: Gastric carcinoma in the Belgian sheperd dog. J Small Anim Pract 32: 465-469

Simpson KW 2010: Diseases of the stomach. In: Ettinger SJ, Feldman EC (eds.): Textbook of veterinary internal medicine. Saunders Elsevier, Canada, pp. 1504-1526

Sullivan M, Lee R, Fischer EW, Nash AS, McCandlish IA 1987: A study of 31 cases of gastric carcinoma in dogs. Vet Rec 120: 79-83

Swann HM, Holt DE 2002: Canine gastric adenocarcinoma and leiomyosarcoma: A retrospective study of 21 cases (1986-1999) and literature review. J Am Anim Hosp Assoc 38: 157-164

Twedt DC 1992: Vomiting. In: Anderson NV, Sherding RG, Merrit AM, Whitlock RH (eds.): Veterinary gastroenterology. Lea \& Febiger, London, pp. 359-361 